\title{
Three-dimensional Working Model of the Multienzyme Aminoacyl-tRNA Synthetase Complex Determined by Computational Microscopy
}

\author{
M.T. Norcum, ${ }^{*}$ C. L. Wolfe, ${ }^{* *}$ and J.A. Warrington* \\ * Biochemistry Department, The University of Mississippi Medical Center, 2500 North State Street, \\ Jackson, MS 39216-4505 \\ ** Biology Department, Tougaloo College, 500 West County Line Road, Tougaloo, MS 39174
}

The aminoacyl tRNA synthetase (aaRS) "core" complex in multicellular eukaryotes is composed of eight proteins with nine enzyme activities and three auxiliary proteins (p43, p38 and p18). Our 2D working model of this particle [1] organizes the proteins into three domains. We now have determined much about the 3D structure of the multi-aaRS complex by calculating reconstructions from electron micrographs using single particle methods [2]. The use of methylamine vanadate (Nanovan) as a negative stain revealed important new structural features [3].

Using this same sample preparation method, we have now identified general sites of tRNA binding using oxidized total yeast tRNA, the active site of LeuRS using Nanogold- labeled tRNA ${ }^{\text {leu }}$, and the C-termini of p43-his 6 using Nanogold-NiNTA. Figures 1 and 2 show typical images used for reconstructions of both control and labeled complexes. Arrows indicate examples of gold-labeled particles. Figure 3 shows that data sets used for projection mapping refinement in order to locate general tRNA binding sites and the LeuRS active site have complete angular coverage and equal representation of views. Fourier shell correlation comparison of reconstructions from half data sets provides resolution limits of $c a .29 \AA$. The data sets and resolution curves for p43 placement are as complete and even as those in Figure 3.

Starting by relating each domain in the 2D model with the basic reconstruction (Figure 4, A and B), we can begin to establish a 3D working model of the aaRS complex. Binding of unfractionated tRNA establishes that most of these molecules are arrayed on the exterior of the structure on all of the domains (Panels C and D, blue areas). Binding of tRNA ${ }^{\text {leu }}$ places LeuRS (large asterisk) and the bifunctional GluProRS (small asterisk) at the base of this asymmetric "V"-shaped multiprotein complex on opposite faces of domain III. The C-termini of p43 are located along one edge of domain II (dark red) and overlap with areas of tRNA binding. The three-domain model places ArgRS in domain II. Evidence that this is appropriate comes from data showing that ArgRS is stabilized within the complex by the N-terminal portion of $\mathrm{p} 43$.

Overall, these results provide the first ideas of the spatial arrangement of proteins, enzyme active sites and multiple substrate binding sites within the multi-aaRS structure. In effect, we now have specific insight into how this unique particle functions as an 'aminoacylation machine'.

References

[1] M.T. Norcum and J.A. Warrington, Prot. Sci. 7 (1998) 79.

[2] J. Frank et al. J. Struct. Biol. 116 (1996) 190.

[3] C.L. Wolfe et al. Prot. Sci. 12 (2003) 2282.

[4] This material is based on work supported by the National Science Foundation under grant numbers MCB-0090539 and MCB-0215940. 

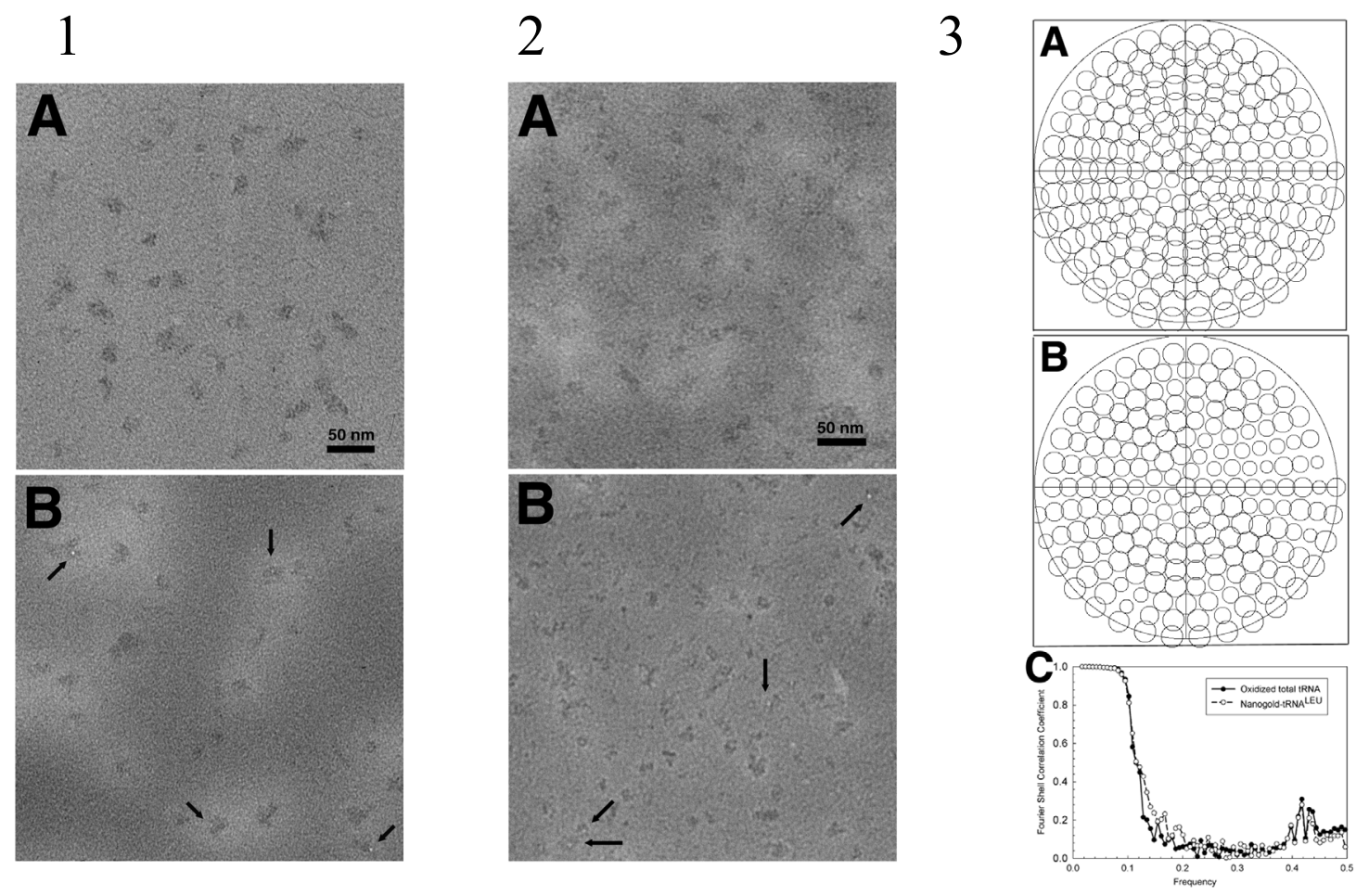

FIG. 1. Typical images used for locating LeuRS. A. Control sample. B. Sample reacted with oxidized Nanogold-tRNA ${ }^{\text {leu }}$.

FIG. 2. Typical images used for locating p43-his 6 . A. Control sample. B. Sample reacted with Nanogold-Ni-NTA

FIG. 3. Demonstration of completeness of data sets and resolution limits for the reconstructions calculated for tRNA-labeled multisynthetase complex. $A$. Angular distribution of images after labeling with total yeast tRNA. $B$. Angular distribution of images after labeling with NanogoldtRNA $^{\text {leu }}$. C. Determination of resolution limits from Fourier shell correlation coefficients.

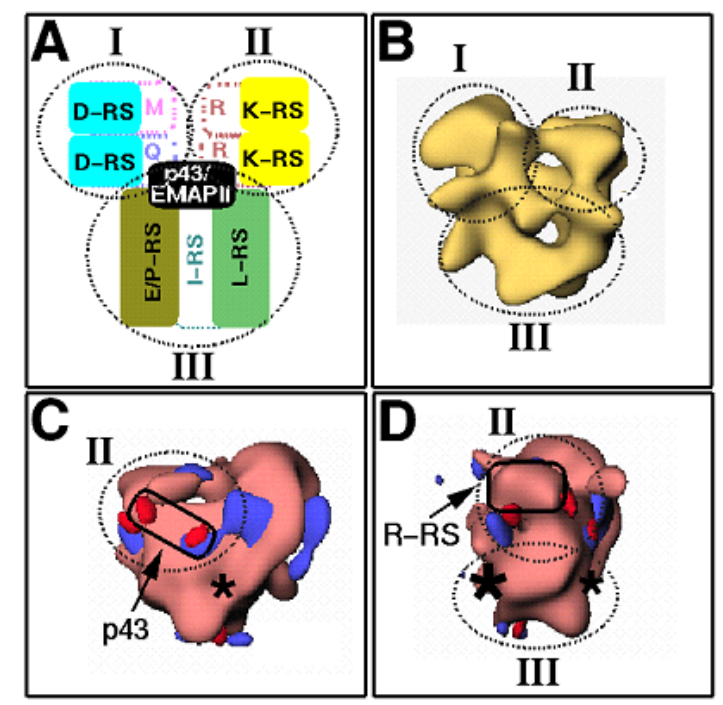

FIG. 4. Extension of the three-domain working model of the multisynthetase complex to three-dimensions. (A) The three-domain model of possible arrangement of proteins within the complex as previously published [1] with the domains delineated by circles. (B) Overlay of the three domains on a "front" view of a surface representation of the three-dimensional reconstruction of the complex [3]. (C) "Back" view with the areas assigned to p43 (dark red) within domain II and its relationship to the position of LeuRS (asterisk) and areas of tRNA binding (blue). (D) "Side" view with the area assigned to ArgRS (solid rectangle) in domain II, as well as the sites of GluProRS (large asterisk) and LeuRS (small asterisk) in domain III. 